PAPER • OPEN ACCESS

\section{Vacuum Polarization and Particle Creation for Two-Horizon Metrics}

To cite this article: M L Fil'chenkov and Yu P Laptev 2021 J. Phys.: Conf. Ser. 2081012018

View the article online for updates and enhancements.
You may also like

- Shadow of Kottler black hole in the
presence of plasma for a co-moving
$\frac{\text { observer }}{\text { Anish Das, Ashis Saha and Sunandan }}$
Gangopadhyay
- High-resolution brain tumor visualization
$\frac{\text { using three-dimensional x-ray phase }}{\text { contrast tomography }}$
F Pfeiffer, O Bunk, C David et al.
- Gauge-invariant description of weak
$\frac{\text { gravitational field on a spherically }}{\text { symmetric background with cosmological }}$
$\frac{\text { constant }}{\text { Piotr Waluk and Jacek Jezierski }}$

The Electrochemical Society

Advancing solid state \& electrochemical science \& technology

243rd Meeting with SOFC-XVIII

Boston, MA • May 28 - June 2, 2023

Accelerate scientific discovery!
Learn More \& Register

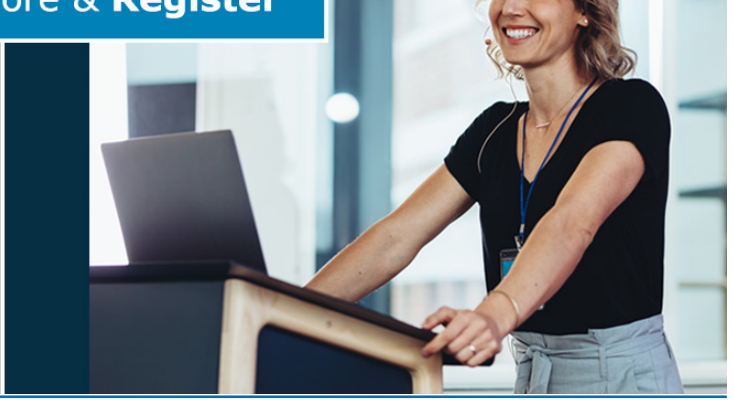

This content was downloaded from IP address 54.224.187.69 on 26/04/2023 at 12:03 


\title{
Vacuum Polarization and Particle Creation for Two-Horizon Metrics
}

\author{
M L Fil'chenkov and Yu P Laptev \\ RUDN University, Moscow, Russia \\ E-mail: fmichael@mail.ru
}

\begin{abstract}
Kerr-Newman and Kottler's metrics with two horizons are considered. Evaporation of Kerr - Newman's horizons in Hawking's effect and Penrose's process as well as de Sitter's horizon decay and Schwarzschild's horizon evaporation for Kottler's metric have been analyzed in terms of an effective temperature, using lifetimes on the horizons. The results are applied to black hole physics and cosmology.
\end{abstract}

\section{Introduction}

The metric horizons result in distortion of the spectrum of vacuum zero oscillations followed by vacuum polarization and particle creation [1]. The presence of horizons is related to a loss of information, rise of entropy and appearance of an effective temperature of vacuum and particles being born. Metrics with two horizons will be considered, viz Kerr - Newman's metric [2] describing a rotating charged black hole and Kottler's cosmological metric [3] for inflation models. Horizon lifetimes determining their evolution have been evaluated.

\section{Metric Horizons, Their Lifetimes. An Effective Temperature on the Horizons}

Kerr - Newman's metric horizons are roots of the equation

$$
\Delta^{2}=r^{2}-\frac{2 G M r}{c^{2}}+\frac{a^{2}}{c^{2}}+\frac{G Q^{2}}{c^{2}}=0,
$$

where $r$ is the radius (in the spherical coordinate system), $M$ the mass, $a$ the specific angular momentum and $Q$ the black hole charge. Since there do not exist massless rotating and / or charged black holes, then the relation

$$
Q^{2}+\frac{a^{2} c^{2}}{G}=\alpha^{2} M^{2},
$$

where $\alpha=$ const, is valid. Kerr - Newman's metric horizons are written in the form:

$$
r_{ \pm}=\frac{G M}{c^{2}}(1 \pm \sqrt{1-A}), A=\frac{a^{2} c^{2}+G Q^{2}}{G^{2} M^{2}}=\frac{a^{2}}{G} \leq 1
$$


where $r_{+}$is the event horizon, $r_{-}$Cauchy's horizon. At $a=0 \quad$ Kerr - Newman's axially symmetric metric reduces to Reissner - Nordström's spherically symmetric metric $[4,5]$ for which $\frac{\Delta^{2}}{r^{2}}=g_{00}$, where $g_{00}$ the temporal component of the metric. For Kottler's metric

$$
g_{00}=1-\frac{r_{g}}{r}-\frac{r^{2}}{r_{0}^{2}}
$$

where $r_{g}$ is Schwarzschild's horizon, $r_{0}$ de Sitter's, in the metrics with horizons enters a surface gravity [6]

$$
\kappa=\frac{c^{2}}{2}\left|g_{00}^{\prime}\left(r_{h}\right)\right|
$$

equal to the acceleration on the horizon $r_{h}$.

The effective temperature on the horizon is given by the formula

$$
k T_{h}=\frac{\hbar \kappa}{2 \pi c} .
$$

The horizon lifetime

$$
\tau_{h}=\int \frac{d E_{h}}{P_{h}},
$$

where $E_{h}$ is the quantity depending on the form of the metric.

$$
P_{h}=4 \pi r_{h}^{2} \sigma T_{h}^{4}
$$

is the power of horizon evaporation or decay,

$$
\sigma=\frac{\pi^{2} k^{4}}{60 \hbar^{3} c^{2}}
$$

Stefan - Bolzmann's constant.

\section{Evaporation of Kerr - Newman's Horizons in Hawking's Effect and Penrose's Process}

Assume that the charge and angular momentum contribute insignificantly to the horizon, i.e. $A=\frac{\alpha^{2}}{G} \ll 1$. Then Kerr-Newman's metric has two horizons:

$$
\begin{gathered}
r_{+}=\frac{2 G M}{c^{2}}=r_{g}, \\
r_{-}=\frac{\alpha^{2} M}{2 c^{2}} .
\end{gathered}
$$

The ratio of these horizon radii

$$
\frac{r_{-}}{r_{+}}=\frac{\alpha^{2}}{4 G} \ll 1
$$


The effective temperatures of the particles born on the horizons:

$$
\begin{gathered}
k T_{+}=\frac{\hbar c^{3}}{8 \pi G M}, \\
k T_{-}=\frac{2 \hbar c^{3} G}{\pi \alpha^{4} M} .
\end{gathered}
$$

The ratio of the temperatures on the horizons

$$
\frac{T_{-}}{T_{+}}=\frac{16 G^{2}}{\alpha^{4}} \gg 1
$$

The energies being lost on the horizons

$$
E_{+}=M c^{2}, E_{-}=\frac{\alpha^{2} M^{2}}{r_{-}}=2 M c^{2} .
$$

From formulae (12), (15) and (16) we obtain:

$$
\frac{\tau_{-}}{\tau_{+}}=\frac{1}{2^{11}}\left(\frac{\alpha^{2}}{G}\right)^{6} \ll 1 .
$$

This means that Cauchy's horizon evaporates considerably faster than the event horizon for a rotating and / or charged black hole, provided that Cauchy's horizon radius is much less than the event horizon one. While evaporating Schwarzschild's horizon in Hawking's effect [7], particles are born. The particles, with a charge opposite to that of the black hole, fall under the horizon diminishing the charge of the latter, which is interpreted as Cauchy's horizon evaporation related as well to a loss of the angular momentum of the black hole due to Penrose's process [8]. Cauchy's horizon evaporation, converts the black hole into Schwarzschild's, which agrees with the results obtained in a manner quite different from ours $[9,10]$.

\section{De Sitter's Horizon Decay and Evaporation of Schwarzschild's for Kottler's Metric}

For $r_{0} \gg r_{g}$ the equation $g_{00}=0$ has two roots $r=r_{g}$ and $r=r_{0}$, which are Schwarzschild and de Sitter's horizons respectively.

The effective temperatures on the horizons:

Schwarzschild's with the effective temperature of particles being created

$$
k T_{H}=\frac{\hbar c}{4 \pi r_{g}},
$$

and de Sitter's with the effective temperature of vacuum being polarized

$$
k T_{d S}=\frac{\hbar c}{2 \pi r_{0}} .
$$

The ratio of the temperature of particles born on Schwarzschild's horizon to de Sitter's vacuum temperature

$$
\frac{T_{H}}{T_{d S}}=\frac{r_{0}}{2 r_{g}} \gg 1 .
$$


Using the quantities

$$
E_{H}=\frac{c^{4} r_{g}}{2 G}, E_{d S}=\frac{4}{3} \varepsilon_{0} r_{0}^{3}=\frac{c^{4} r_{0}}{2 G}
$$

where

$$
\varepsilon_{0}=\frac{3 c^{4}}{8 \pi G r_{0}^{2}}
$$

is de Sitter's vacuum energy density, from formulae (20) - (22) we obtain the ratio of de Sitter's horizon lifetime to that of Schwarzschild's:

$$
\frac{\tau_{d S}}{\tau_{H}}=\frac{1}{2^{4}}\left(\frac{r_{0}}{r_{g}}\right)^{3} \gg 1 .
$$

This means that Schwarzschild's horizons of supermassive black holes at the centres of galaxies evaporate faster than de Sitter's horizon of dark energy, which is a well-known result [11]. However, in case

$$
\frac{\tau_{d S}}{\tau_{H}}<1,
$$

if

$$
\frac{r_{0}}{r_{g}}<2^{4 / 3}
$$

i. e. de Sitter's horizon in the early Universe decays before the evaporation of primordial black holes.

\section{Conclusion}

The evaporation of Cauchy's horizon before the event one of Kerr - Newman's metric proceeds due to a loss of mass, charge and angular momentum in Hawking's effect and Penrose's process. De Sitter's horizon decay and Schwarzschild's horizon evaporation for Kottler's metric are due to particle creation after the first inflation in the early Universe (Big Bang) and the evaporation of massive black holes in a more stable de Sitter vacuum before the completion of the second inflation (dark energy).

\section{References}

[1] Grib A A, Mamaev S G and Mostepanenko V M 1994 Vacuum Quantum Effects in Strong Fields (St. Petersburg: Friedmann Lab. Publ.)

[2] Newman E N et al 1965 J. Math. Phys. 6918

[3] Kottler F 1918 Ann. Phys. 56401

[4] Reissner H 1916 Ann. Phys. 5015

[5] Nordström G 1918 Proc. Kon. Ned. Akad. Wt. 201238

[6] Gibbons G W, Hawking S W 1977 Phys. Rev. D 152738

[7] Hawking S W 1975 Comm. Math. Phys. 43199

[8] Penrose R 1969 Nuovo Cim. 1252

[9] Gal'tsov D V 1986 Particles and Fields in the Vicinity of Black Holes (Moscow: Moscow University Press)

[10] Novikov I D, Frolov V P 1986 Black Hole Physics (Moscow: Nauka, Fizmatlit)

[11] Grib A A, Pavlov Yu V 2016 Grav. Cosmol. 22107 


\section{Acknowlegements}

This publication was prepared with the support of the "RUDN University Program 5-100". 